\title{
Mouse cytokine profile skewed towards Th2 in pregnancy during infection with Brucella abortus S19 strain
}

\author{
FRANCIS O. WAMONJE1* ${ }^{*}$, REBECCA K. WAIHENYA ${ }^{1}$, ZIPPORAH NG'ANG'A ${ }^{2}$ and JOSEPH \\ N. NGERANWA ${ }^{3}$ \\ ${ }^{1}$ Department of Zoology, Jomo Kenyatta University of Agriculture and Technology, P.O. Box 62000- \\ 00200, Nairobi, Kenya \\ ${ }^{2}$ Institute of Tropical Medicine and Infectious Diseases, Jomo Kenyatta University of Agriculture and \\ Technology, Nairobi, Kenya \\ ${ }^{3}$ Department of Biochemistry and Biotechnology, Kenyatta University, Nairobi, Kenya
}

\begin{abstract}
The two classes of cytokines Th1 and Th2 determine the type of immune response elicited. The Th2 immune response is associated with successful pregnancy. Brucellosis is an intracellular bacterium that elicits the Th1 response and is known to cause spontaneous abortion in mammalian species. This study sought to determine if Brucella infection causes spontaneous abortion by causing the circulating cytokine profile be Th1 dominant during pregnancy. Fortyeight Swiss white mice were used in this murine model and the S19 strain of Brucella abortus was used in as the infective agent. Pregnant mice in the test group were injected intraperitoneally with 105-8 CFU of Brucella and cytokine profile evaluated over the three trimesters of pregnancy. Pregnant mice in the control group were left to go through normal pregnancy and their cytokine profile evaluated over the three trimesters of pregnancy. Cytokines in serum samples were analyzed by Cytometric Bead Array. The data was analyzed using the Paired T- test and $\mathrm{p}<0.05$ was considered significant. IFN- $\gamma$ and TNF- $\alpha$ represented the Th1 cytokines while IL-4 and IL-5 represented the Th2 cytokines. None of the mice in the test group had spontaneous abortion. IFN- $\gamma$ and TNF- $\alpha$ had no significant differences between cytokine levels for infected and uninfected groups in all 3 trimesters of pregnancy. IL-4 levels had significant differences in all three trimesters of pregnancy $(t=13, P=0.036,0.0071$ and 0.0277 ). IL-5 levels had significant differences second trimester $(\mathrm{t}=14, P=0.0075)$. The cytokine profile was robustly Th2. In conclusion, Brucella abortus cannot cause spontaneous abortion by altering the mouse cytokine profile towards ThI in pregnancy. Elevated IL-4 levels with corresponding suppression of IFN- $\gamma$ can be used as a marker for successful pregnancy in Brucellosis.
\end{abstract}

Key words: Cytokines, Brucella, Interleukin, Interferon, T-helper 1, T-helper 2

\section{Introduction}

Brucellosis caused by the bacteria Brucella is the most common zoonotic disease in the world (Rust, 2006). The disease has been implicated in causing spontaneous abortions in animals and man (Khan et al., 2001). Spontaneous abortion is the loss of the uterine product prior to the viability of the foetus (Raghupathy, 1997). When this occurs repeatedly, the condition is referred to as repeated spontaneous abortion (RSA). Pathogenic strains possess a non-endotoxic lipopolysaccharide that confers resistance to anti-microbial attacks and modulates the host immune response (Cardoso et al., 2006). Clinical and experimental evidence has indicated that the maternal immune response is

* Correspondence: Francis O. Wamonje; E-mail: francisonono@gmail.com 
biased toward a antibody mediated T-helper 2 (Th2) response during pregnancy (Lin et al., 1993; Robertson et al., 1994; Raghupathy, 1997; Pankaj, 2007; Gagandeep et al., 2008). The T-helper 1 (Th1) immune response is cell mediated and mainly inflammatory and is considered incompatible with successful pregnancy (Raghupathy, 1997). The Th1 response's primary trigger is Interferon- gamma (IFN- $\gamma$ ) which induces a bactericidal effect by natural killer cells and $\mathrm{T}$ lymphocytes directly and through macrophage induction (Celli, 2006). In vitro treatment of macrophages with recombinant IFN- $\gamma$ has been shown to enhance killing of Brucella abortus and in vivo experiments showed enhanced resistance to B. abortus and Listeria monocytogenes in mice (Kamruddin et al., 1999). TNF-alpha (TNF- $\alpha$ ) plays a central role in activation of first-line defences of a host against foreign organisms. Brucella has been shown to modulate TNF- $\alpha$ production as an immune evasion strategy within the host (Caron et al., 1994). The main trigger for the Th2 response is IL-4. IL-4 is known to be antagonistic to IFN- $\gamma$ and has been shown to up regulate class II MHC molecules. This in effect enhances activation of antibody presenting cells involved in the generation of antimicrobial or allotypic immune responses in pregnancy (Moraes- Pinto et al., 1998). By examining the cytokine profile in pregnancy, the objective of this study was to establish if Brucella can elicit a dominant Th1 response in pregnancy and therefore ascertain if this is method by which it causes spontaneous abortion.

\section{Materials and Methods}

\section{Experimental animals and inoculation with Brucella}

Swiss white mice were used, 48 female and 12 male mice were obtained from the University of Nairobi School of Biological Sciences animal house. Mice were weighed upon arrival to ascertain if they had acquired an adult weight of $>20$ gms. For this experiment, a weight of 25 grams and above was desirable. The animals were penned for a week to acclimatize to their new environment. They were allowed unlimited access to food pellets and water on a twelve hour light- dark cycle. Brucella abortus S19 strain was used. This was obtained from Ondestepoort Veterinary Institute- South Africa.

There were four (4) mice for both the test and control groups in each trimester of pregnancy making a total of twelve (12) mice for each group. Four female mice were penned alongside one adult male. Formation of a vaginal plug was indicative of successful mating. Observation of the plug was indicated as Day 0.5 of pregnancy. The critical days were days 3.5, 9.5 and 15.5 which were mid first, second and third trimester respectively. Mice were inoculated intraperitonealy with approximately $10^{5-8} \mathrm{CFU}$ of Brucella in a $1.0 \mathrm{ml}$ suspension.

\section{Blood collection from mice for cytokine analysis}

Blood was collected by cardiac puncture using a $29 \mathrm{G}$ needle. On average $0.15 \mathrm{ml}$ of blood yielded sufficient serum. Blood was collected into non heparinised plain tubes and allowed to settle. Serum was extracted using a micropipette, transferred into labelled Cryo tubes and stored at $-80^{\circ} \mathrm{C}$ until use. Blood was collected on days $3.5,9.5$ and 
15.5 which represented the three trimesters of pregnancy. This experiment was carried out in two replicates. A total of 48 serum samples were collected from both replicates.

\section{Laboratory analysis of serum samples for cytokine levels}

All samples were analyzed by flow cytometry using the Cytometric Bead Array (CBA). The protocol for analysis is designed by BD biosciences that are the manufactures of the reagent. Fifty micro litres of the mixed Capture Beads were vortexed and then added to the appropriate assay tubes. A $50 \mu \mathrm{l}$ of the Mouse Th1/Th2 Cytokine Standard dilutions were then added to the control assay tubes. Fifty micro litres of each test sample was measured into the test assay tubes and $50 \mu \mathrm{l}$ of the Mouse Th1/Th2 PE Detection Reagent added to the assay tubes. The assay tubes were then incubated for 2 hours at room temperature protected from direct exposure to light. $1 \mathrm{ml}$ of Wash Buffer was added to each assay tube and centrifuged at $200 \times \mathrm{g}$ for 5 minutes. The supernatant was carefully aspirated and discarded from each assay tube. $300 \mu \mathrm{l}$ of Wash Buffer was be added to each assay tube to re-suspend the bead pellet. The samples were now ready for analysis on a flow cytometer. Each sample was vortexed for 3 - 5 seconds immediately before analyzing on the flow cytometer. Cytokine levels were measured from standard curves calibrated from reagent standards using the flow cytometer's software and the values were in $\mathrm{pg} / \mathrm{ml}$.

\section{Data analysis}

Readings falling below the detectable threshold $(\leq 0)$ were disregarded in the statistical analysis. Data was analyzed using the using EXCEL statistical software. The test and control groups were tested for homosedasticity in the variances using the F-test and were found to have statistically significant differences. The un-paired t-test assuming unequal variances was then used to test significance between the means of the groups at 95\% confidence level.

\section{Results}

No spontaneous abortions were seen or recorded during all the three trimesters of pregnancy for all the mice. Cytokine levels were measured for the three trimesters of pregnancy on days 3.5, 9.5 and 15.5. The mean cytokine readings for test and control groups for the three trimesters of pregnancy are shown in Tables 1, 2 and 3.

Table 1: Mean cytokine measurements for test and control groups mid first trimester of pregnancy

\begin{tabular}{llll}
\hline Cytokine & $\begin{array}{l}\text { Test group (Infected) } \\
\mathbf{p g} / \mathbf{m l} \mathbf{( M} \pm \mathbf{S D})\end{array}$ & $\begin{array}{l}\text { Control group (Uninfected) } \\
\mathbf{p g} / \mathbf{m l} \mathbf{~ ( M \pm ~ S D )}\end{array}$ & P-value \\
\hline IFN- $\gamma$ & $3.03 \pm 2.31$ & $4.70 \pm 4.74$ & 0.30 \\
TNF- $\alpha$ & $10.43 \pm 22.63$ & $2.42 \pm 0.92$ & 0.35 \\
IL-4 & $189.02 \pm 226.35$ & $3.43 \pm 3.24$ & 0.053 \\
IL-5 & $16.34 \pm 14.15$ & $8.29 \pm 3.87$ & 0.15 \\
\hline
\end{tabular}


In the first trimester, differences between mean cytokine levels between control and test groups were not significant for IFN- $\gamma$; t (8), $\mathrm{p}=0.30$, TNF- $\alpha$; $\mathrm{t}(14), \mathrm{p}=0.35$ and IL-5; $\mathrm{t}(14)$, $\mathrm{p}=0.15$. Mean IL-4 levels were markedly higher between the test and control and were statistically significant IL-4; $\mathrm{t}(14) \mathrm{p}=0.053(P<0.05)$

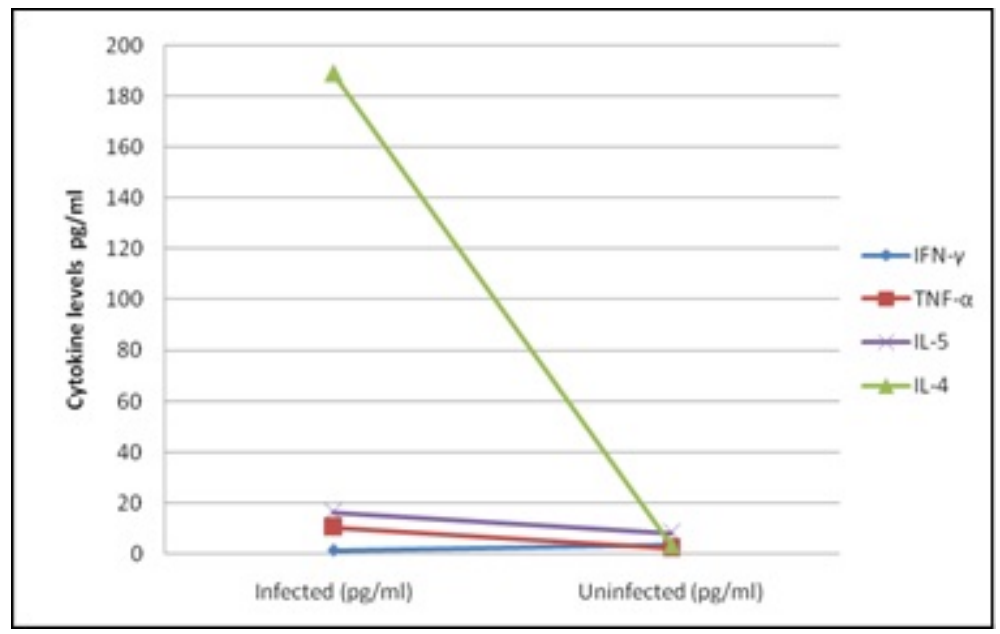

Figure 1: First trimester cytokine levels

Cytokine levels for the infected group showed IL-4 followed afar by IL- 5 were the most dominant cytokines in circulation. IFN- $\gamma$ levels were lowest followed by those of TNF$\alpha$. The uninfected group did not show much variation in the cytokine levels.

Table 2: Mean cytokine measurements for test and control groups mid second trimester of pregnancy

\begin{tabular}{llll}
\hline Cytokine & $\begin{array}{l}\text { Test group (Infected) } \\
\text { Pg/ml (M } \pm \mathbf{~ S D )}\end{array}$ & $\begin{array}{l}\text { Control group (Uninfected) } \\
\mathbf{p g} / \mathbf{m l} \mathbf{( M} \pm \mathbf{S D})\end{array}$ & P- value \\
\hline IFN- $\gamma$ & $2.34 \pm 1.4$ & $6.43 \pm 5.47$ & 0.47 \\
TNF- $\alpha$ & $2.41 \pm 0.34$ & $2.27 \pm 0.53$ & 0.54 \\
IL-4 & $37.39 \pm 22.61$ & $2.5 \pm 7.79$ & 0.003 \\
IL-5 & $19.94 \pm 11.78$ & $6.7 \pm 1.56$ & 0.016 \\
\hline
\end{tabular}

In the second trimester, differences between mean cytokine levels between control and test groups were not significant for IFN- $\gamma$; $\mathrm{t}(4), P=0.47$ and TNF- $\alpha$; $\mathrm{t}(14), P=0.54$. Mean IL-4 levels had dropped in both the test group and control group but the difference was still statistically significant. IL-4; $\mathrm{t}(13), P=0.003$. Mean IL-5 levels rose in test group and dropped in the control group. This difference was statistically significant.

IL-5; t (14), $P=0.016$.

Cytokine levels for IL-4 dropped but it was still the most dominant cytokine in circulation followed by IFN- $\gamma$ levels remained lowest and there was a corresponding drop in TNF- $\alpha$. The uninfected group did not show much variation in the cytokine levels. 


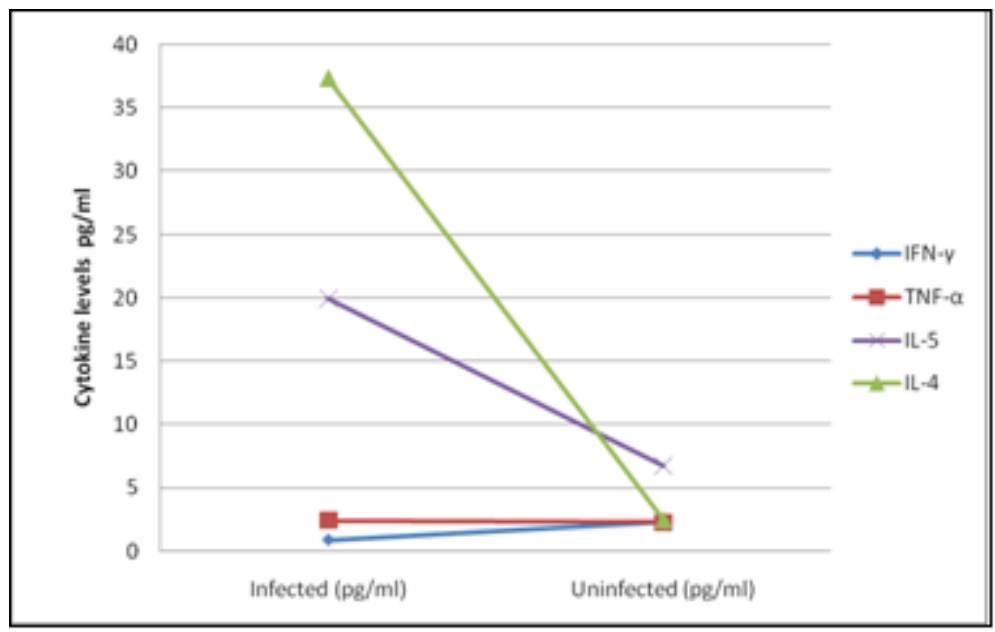

Figure 2: Second trimester cytokine levels

Table 3: Mean cytokine measurements for test and control groups mid third trimester of pregnancy

\begin{tabular}{llll}
\hline Cytokine & $\begin{array}{l}\text { Test group (Infected) } \\
\mathbf{p g} / \mathbf{m l} \mathbf{( M} \pm \mathbf{S D})\end{array}$ & $\begin{array}{l}\text { Control group (Uninfected) } \\
\mathbf{p g} / \mathbf{m l} \mathbf{( M} \pm \mathbf{S D})\end{array}$ & P value \\
\hline IFN- $\gamma$ & $3.27 \pm 1.90$ & $9.35 \pm 6.99$ & 0.21 \\
TNF- $\alpha$ & $2.64 \pm 1.10$ & $2.86 \pm 0.97$ & 0.67 \\
IL-4 & $17.66 \pm 12.02$ & $5.91 \pm 4.32$ & 0.014 \\
IL-5 & $12.69 \pm 3.22$ & $12.67 \pm 6.46$ & 0.54 \\
\hline
\end{tabular}

Differences between mean cytokine levels between control and test groups were not significant for IFN- $\gamma$; t (8), $P=0.21$ TNF- $\alpha$; t (14) $P=0.67$ and IL-5 t (14) $P=0.54$ Despite the drop in mean IL-4 levels for the test group and a transient increase in the control group the differences in the means remained statistically significant IL-4; $\mathrm{t}(14), P=0.014$ in the third trimester of pregnancy.

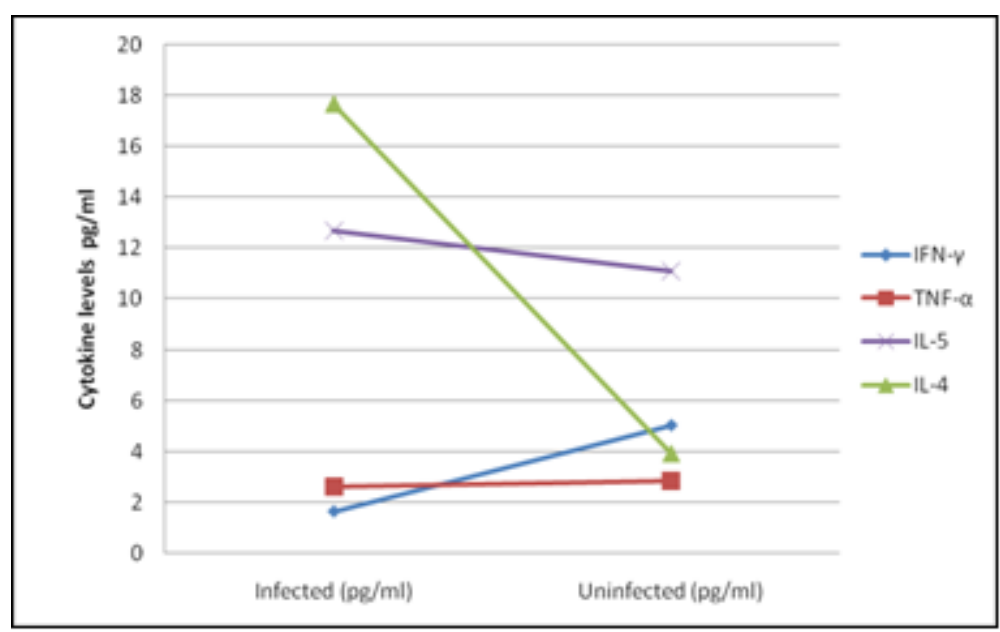

Figure 3: Third trimester cytokine levels 
IL-4 and IL-5 maintained their stranglehold as the main cytokines in circulation in the infected group. IFN- $\gamma$ gained more prominence in the uninfected group.

\section{Discussion}

There were no significant differences in the Th1 cytokines; IFN- $\gamma$ and TNF- $\alpha$ between the control and test mice during all three trimesters of pregnancy in this study. IFN- $\gamma$ levels in the test group were low in the first trimester. IFN- $\gamma$ and IL-12 are the main triggers for differentiation of the Th0 cell into Th1 subtypes. The classical immunologic effect of IFN- $\gamma$ is macrophage activation and promotion of Th1- differentiation thereby creating a cellular immune response. This study showed suppression of IFN- $\gamma$ in the test group this in effect led to the stifling of the Th1 immune response. These results differed with those of Suk et al. (2005), where upon challenge with B. abortus; they observed an increase in IFN- $\gamma$ during the first trimester of pregnancy implicating it in causing spontaneous abortion. During the acute phase of infection of bacterial infection IFN- $\gamma$ is most active. Of the four cytokine levels measured, it was lowest. Since its immunomodulatory actions are considered most detrimental to successful pregnancy, its low levels account for the normalcy in pregnancy during this period when spontaneous abortion would have been expected. IFN- $\gamma$ was markedly suppressed in the infected group and this could be attributed to the antagonistic action of IL-4. The transient increase in the third trimester could be due to the lower level of IL-4 in circulation though it is not known at what level IL-4 stops being antagonistic to IFN- $\gamma$. The uninfected (control) group levels were higher at all times than the test group. IFN- $\gamma$ was in near equilibrium with IL-4 during the entire pregnancy suggesting a delicate balance between these cytokines during the entire pregnancy.

There were higher levels of TNF- $\alpha$ in the Brucella infected mice during the first trimester of pregnancy however, the levels were not significant. In this study, TNF- $\alpha$ level in the infected (test) group were highest when measured mid first trimester, dropped sharply by mid second trimester and rose transiently in the final trimester. The uninfected (control) group levels were consistently low and showed little change during all trimesters of pregnancy. Pregnancy is also known not to favour production of TNF- $\alpha$ (Sidoti et al., 1998). Also other studies have shown a down regulation of TNF- $\alpha$ during pregnancy when challenged with bacterial lipopolysaccharide (Bouman et al., 2003). This is the scenario that brucellosis presents and this can explain the drop in TNF- $\alpha$ level between the first and second trimesters in this study despite stimulation with Brucella bacterial LPS. Brucella infection uses pathways that avoid TNF- $\alpha$ and actively inhibit its production as a major attribute of its virulence. The initial high levels of TNF- $\alpha$ seen in the infected group when compared to the uninfected group suggest that this initial spike was due to Brucella infection and the subsequent drop attributed to the inhibition of TNF- $\alpha$ by Brucella bacteria. In this study, inhibition of TNF- $\alpha$ as an immune evasion mechanism was evidenced by the rapid drop in TNF- $\alpha$ level and is consistent with findings by both Caron et al. (1994) and Pappas et al. (2005).

There was significant difference in the cytokine levels for IL-4 control and test groups in all three trimesters of pregnancy. IL-4 is the main cytokine involved in 
modulating the Th2 response and also promotes the proliferation of other Th2 cells by inhibiting the Th1 immune response (Nelms et al. 1999). The differences in the levels between infected and uninfected groups were explicit in showing the robustness on the Th2 response and especially in the first trimester of pregnancy. For this study, this was critical as this represented the acute phase of the infection. The experiment was able to dramatically capture the impact of the humoral response led by IL-4 on the Th1 cytokines. The high level of IL-4 in circulation is of importance, because IL- 4 is the Th2 cytokine with most antagonistic effects to IFN- $\gamma$ which at excessive levels is believed to be detrimental in pregnancy. In this study, IL-4 levels were elevated at the expense of IFN- $\gamma$ consistent with the observations by Paludan (1998) and Ekerfelt et al. (2002). Normal function of IFN- $\gamma$ in response to intracellular bacteria was suppressed and this can be directly attributed to the high level of IL-4. It is therefore clear why there were no spontaneous abortions during this experiment. All the mice were able to carry the pregnancies successfully to full term. The significant difference between the control and test group in all trimesters suggests that the pregnant mice produce IL- 4 in response to intracellular bacterial infection. These results were consistent with those of Leif (1998) and Moraes- Pinto (1998). In the second trimester IL-4 levels dropped sharply (Figure 2) from as expected since Brucella S19 strain is rapidly cleared from circulation (OIE, 2008). Rapid clearance from circulation will cause less MHC class II stimulation and subsequent down regulation if the immune response.

Not much is known about IL-5 profile in Brucella infection. There was significant difference in the cytokine levels for IL5 control and test groups during the second trimester. Test group IL-5 levels rose significantly suggesting a deliberate shift by the maternal immune system towards a humoral configuration. This suggests synergism between IL-4 and IL-5 in propagation of the humoral response probably due to the close proximity of genes that code for these two cytokines. This finding was consistent with a study by Rezazadeh et al. (2006).

No spontaneous abortions were seen during this study. The results correctly showed that the Th2 profile is dominant in pregnancy even in the presence of intracellular bacteria such as Brucella that would naturally cause high production of Th1 cytokines. IFN- $\gamma$ production is suppressed as an antagonistic action of IL- 4 while TNF- $\alpha$ is suppressed as an immune evasion mechanism of Brucella bacteria. Throughout all three trimesters of pregnancy, the Th1 cytokines remained suppressed. This experiment's results are consistent with the research done by Lin et al. (1993), Robertson et al. (1994), Raghupathy (1997) and Pankaj (2007) that the maternal immune response is skewed towards Th2 in pregnancy.

In conclusion, the Brucella abortus S19 strain used did not cause spontaneous abortion in this experiment. The maternal cytokine profile was not altered towards a Th1 response that would have favoured spontaneous abortion. A strong humoral response was elicited by the mice during pregnancy and this immunomodulatory action was mediated through IL-4 suppressing IFN- $\gamma$ hence protecting the foetus from the harmful effects of a cellular response. Since IFN- $\gamma$ and IL- 4 have been used as pro and antiinflammation markers of disease progression respectively, elevated levels of IL-4 with a corresponding drop in IFN- $\gamma$ levels can be used as indicators of a favourable prognosis 
in pregnancy. There is need for development and promotion of usage of human vaccines against brucellosis especially those that elicit a strong Th2 response. Vaccines developed should target the enhancement of the Th2 response during pregnancy as a counter to recurrent spontaneous abortions. The major limitation of this research is that the cytokine profile presented in this study is limited to the S19 strain only.

\section{Acknowledgements}

The first author is greatly indebted to Mrs. R.A. Onono for sponsoring this work. A lot of thanks to Mrs. Mercy Njoki Gitau of Zoology Department for her expertise in handling the animals and Mr. Anderson Wambugu from the National Agriculture Research Laboratories- Kenya Agriculture Research Institute, for his sound advice on Brucella. The author would like thank Mr. Kellern Wafula from BD and Loise Munyambu from Faram EA Ltd. for the reagents and technical input for Cytometric Bead Array. Mr. Franklin Onchiri from the University of Nairobi is acknowledged for his assistance in the statistical analysis.

\section{References}

Bouman, A., Moes, H., Heineman, M.J., De Leij, L.F.M.H. \& Faas, M.M. (2003) Endotoxin-induced cytokine production of monocytes of third trimester pregnant women compared with women in the follicular phase of the menstrual cycle. American Journal of Obstetrics \& Gynecology 188, 1073-1077.

Cardoso, P.G., Marcedo, G.C., Azevedo, V. \& Oliveira, S.C. (2006) Brucella spp noncanonical LPS: Structure, biosynthesis and interaction with the immune system. Microbial Cell Factories 5, 13.

Caron, E., Peyrard, T., Köhler, S., Cabane, S., Liautard, J.P. \& Dornand, J. (1994) Live Brucella spp. fail to induce tumor necrosis factor alpha excretion upon infection of U937-derived phagocytes. Infection and Immunity, 62, 5267-5274.

Celli, J. (2006) Surviving inside a macrophage: The many ways of Brucella. Research in Microbiology 157, 93-98.

Ekerfelt, C., Lidstroèm, C., Matthiesen, L., Berg, G., Sharma, S. \& Ererudh, J. (2002) Spontaneous secretion of interleukin- 4 , interleukin-10 and interferon- $\gamma$ by first trimester decidual mononuclear cells. American Journal of Reproductive Immunology 7,159-166.

Gagandeep, K.W., Rupak, M., Saraswathy, K.N., Manju, P. \& Chahal1, S.M.S. (2008) Immuno-molecular etiology of recurrent pregnancy loss and the anthropological perspective. International Journal of Human Genetics 8, 227-235.

Kamruddin, A., Kismat, A.A., Glenda, M., Kazunori, O., Vincent, O.R. \& Tsuyoshi, N. (1999) Increased serum levels of interferon- $\gamma$ and interleukin-12 during human brucellosis. American Journal of Tropical Medicine and Hygiene 61, 425-427.

Khan, M.Y., Mah, W.M., and Memish, Z.A. (2001) Brucellosis in pregnant women. Journal of Clinical Infectious Diseases 32, 1172- 1177. 
Leif, S.M. (1998) Immune Changes in Pregnancy: A Survey of some Immunological Variables in Normal and Complicated Pregnancies. Lonkoping University medical dissertation No. $253 . \quad$ Available from: http://www.bibl.liu.se/liupubl/disp/disp98/med563s.htm (Accessed on 18 June 2009).

Lin, H., Mossman, T.R., Guilbert, L., Tuntipopipat, S. \& Wegmann, T.G. (1993) Synthesis of T helper 2-type cytokines at the maternal-fetal interface. Journal of Immunology 151, 4562-4573.

Moraes-Pinto, M.I., Vince, G.S., Flanagan, B.F., Hart, C.A. \& Johnson, P.M. (1998). Localization of IL4 and IL4 receptors in the human term placenta, decidua and amniochorionic membranes. Immunology 90, 87-94.

Nelms, K., Keegan, A.D., Zamorano, J., Ryan, J.J. \& Paul, W.E. (1999) The IL-4 Receptor: Signaling Mechanisms and Biologic Functions. Annual Review of Immunology 17, 701-738.

OIE (2008) Bovine Brucellosis. Terrestrial Manual 624-659.

Paludan, S.R. (1998) Interleukin 4 and Interferon $\gamma$ : The essence of a mutually antagonistic relationship. Scandnavian Journal of Immunology 48, 459- 468.

Pankaj, D. (2007) Cytokines in Obstetrics and Gynaecology. The Journal of Obstetrics and Gynecology of India 57, 205-209.

Pappas, G., Akritidis, N., Bosilkovski, M. \& Tsianos, E. (2005) Brucellosis. New England Journal of Medicine, 352 (22): 2325-2336.

Raghupathy, R. (1997) Th1-type immunity is incompatible with successful pregnancy. Immunology Today, 18, 478-482.

Rezazadeh. M., Hajilooi, M., Haidari, M., Rafiei, A., Alavi, S.A. \& Keramat, F. (2006) Association of susceptibility to brucellosis and interleukin-4 promoter polymorphism. Scandinavian Journal of Infectious Diseases 38, 1045-1049.

Robertson, S.A., Seamark, R.F., Guilbert, L.J. \& Wegmann, T.G. (1994) The role of cytokines in gestation. Critical Review of Immunology 14, 239-292.

Rust, R.S. (2006) Brucellosis. e-Medicine Journal. http://emedicine.medscape.com/article/1164632-overview (Accessed 12 March 2007)

Suk, K., Dong, S.L., Kenta, W., Hidefumi, F., Hiroshi, S. \& Masahisa, W. (2005) Interferon $\gamma$ promotes abortion due to Brucella infection in pregnant mice. Microbiology 5, 22-29. 